\title{
Enfermedad de Moyamoya: Cefalea crónica y demencia progresiva asociadas a infartos cerebrales múltiples
}

\author{
Moyamoya disease: Chronic headache and progressive dementia associated with multiple brain strokes
}

Marco A. Castañeda ${ }^{1,2}$, Carlos Saul Peña ${ }^{2,3}$, Medalit Liliana Lucho Cabillas ${ }^{4}$

RESUMEN

Se reporta el caso de un paciente de 32 años de edad que desde los 17 y hasta la actualidad, ha experimentado cefaleas constrictivas de minutos de duración, desencadenadas por actividad o esfuerzos físicos. En los últimos siete años ha presentado progresiva declinación cognitiva y cambios acentuados de personalidad con creciente dependencia para el desarrollo de actividades elementales. A los 27 años desarrolló hemiplejía izquierda total con recuperación completa en dos semanas. Muestra asimismo episodios de estado confusional severo y conducta infantil. Los exámenes de laboratorio descartaron cardiopatía, ateroesclerosis y enfermedades autoinmunes sistémicas en el paciente. Estudios neuroimagenológicos del cerebro (TAC, Angio - TEM y RM cerebralcontrastada) pusieron en evidencia seis infartos cerebrales, en estadios subagudos y crónicos, severa estenosis de las arterias cerebrales anteriores y presencia de un microaneurisma en el segmento M de la arteria cerebral media izquierda. Una limitación del presente reporte es la ausencia de estudios con angiografía digital, debida a dificultades económicas. En conclusión, el caso corresponde a un paciente con cefalea crónica asociada a demencia progresiva, en el que estudios de neuroimágenes y pruebas neuropsicológicas sustentan el diagnóstico de deterioro cognitivo asociado a la enfermedad de Moyamoya.

PALABRAS CLAVE: Enfermedad de Moyamoya, demencia, infartos múltiples.

\section{SUMMARY}

The case of a 32 years old. male patient who since age 17 has experienced constrictive headaches of several minutes' duration, triggered by physical effort is reported. For the last seven years there, he has presented a progressive cognitive decline cognitive, and marked personality changes, having become extremely dependent even for conducting elemental activities. At the age of 27 , he experienced total left hemiplegia from which he completely recovered in two weeks. He also shows severe confusional states and child-like behavior. Laboratory tests ruled out heart disease, atherosclerosis and systemic autoimmune diseases. Brain neuroimaging tests (CT, Angio-TEM and contrasted cerebral MRI) revealed the occurrence of six cerebral infarcts, in subacute and chronic stages, severe stenosis of the anterior cerebral arteries, and presence of microaneurysm in the M segment of the left middle brain artery. A limitation of this report is the absence of a study with digital angiography, due to economic difficulties. In short, the case of a patient with chronic headache associated with progressive dementia is presented, with neuroimaging and neuropsychological tests that support the diagnosis of of cognitive impairment associated with Moyamoya disease.

KEY WORDS: Moyamoya disease, dementia, multiple heart attacks.

\footnotetext{
Servicio de Neurología, Hospital Nacional Dos de Mayo. Lima, Perú.

Universidad Nacional Mayor de San Marcos. Lima, Perú.

Servicio de Radiología, Hospital Nacional Dos de Mayo. Lima, Perú.

Servicio de Psicología, Hospital Nacional Dos de Mayo. Lima, Perú.
} 


\section{INTRODUCCION}

La enfermedad de moyamoya (EMM) es una enfermedad cerebrovascular oclusiva caracterizada por estenosis progresiva o la oclusión en la porción terminal de las arterias carótidas internas bilaterales, que afectan tanto a niños como a adultos. Descrita por primera vez en Japón por Takeuchi y Shimizu en 1957(1). Aunque es más común en Japón, se ha detectado más casos clínicos de esta patología en otros lugares del mundo. Afecta con mayor frecuencia a niños de 5 años y adultos menores de 40 años. Hay casi el doble de pacientes mujeres que hombres (2). La etiología de la enfermedad no está clara, aunque los hallazgos de mayor incidencia están en Asia oriental y el hecho de que esta patología es con frecuencia familiar sugieren la implicación de un factor genético en su patogénesis, e inclusive se ha descrito un patrón de herencia autosómica dominante de penetrancia incompleta en familias japonesas (3). Las manifestaciones clínicas varían entre regiones geográficas. Los estudios que involucran poblaciones asiáticas indican que los adultos tienen tasas de hemorragia muchos más altas como síntoma de presentación (42\%) que los adultos estadounidenses (4). En contraste, sólo el $2,8 \%$ de los niños en poblaciones asiáticas presentan hemorragias y el $68 \%$ padecen ictus isquémicos (5). La investigación de los cambios producidos en los vasos arteriales en la bifurcación de las carótidas o la arteria cerebral media proximal, consistentes en estenosis severas, hemorragias y la presencia de colaterales por el defecto de perfusión (vasos moyamoya) requiere del empleo de TEM, Angio-TEM y RM que permiten observar los sucesos isquémicos y/o hemorrágicos. Es el estudio angiográfíco con la sustracción digital, el más indicado para poder objetivar los vasos moyamoya "nubes de humo". El uso reciente de técnicas de RM de alta resolución ha posibilitado un avance en el comportamiento encéfalo - vascular de la enfermedad (6), que permite diferenciar las diferentes causas de estenosis arterial.

Presentamos los datos clínicos, los resultados de laboratorio y los hallazgos de neuroimágenes, La exploración neuropsicológica fue obligatoria y permitió precisar las características del deterioro cognitivo del paciente, considerando la cronicidad de sus trastornos y los trastornos de la personalidad asociados a patología vascular sugerentes de enfermedad de "moyamoya".

\section{Descripción del caso}

Paciente de 32 años de edad, natural de Lima, estudió hasta el sexto grado de primaria, con pobre rendimiento escolar, trabajó como ayudante automotriz en negocio informal de reparación automotriz . Padece de episodios de cefalea desde los 17 años de edad.

$\mathrm{Su}$ conviviente informó que el paciente padece de crisis intensas de dolor de cabeza desde la edad de 17 los diecisiete años, con una frecuencia de tres crisis por mes con localización occipital, con una frecuencia aproximada de 3 crisis por mes de severa intensidad severa intensidad y discapacitante ; desencadenadas por actividades de esfuerzo. En los últimos siete años, en forma gradual y progresiva se han producido cambios cognitivos y conductuales progresivos consistentes en apatía, aislamiento social, en inatención, disturbios de la memoria y concentración, descuido personal en el vestir y el aseo, indiferencia afectiva, desmotivación para actividades del trabajo, apatía y aislamiento social. Hace cinco años que desarrolló hemiplejía derecha sin disfasia, consultó en el Hospital "Guillermo Almenara Irigoyen", tuvo el diagnóstico de infarto subcortical izquierdo, mediante estudio con TAC cerebral. El tratamiento farmacológico y la fisioterapia permitió su recuperación en 2 semanas.

No se precisó de la etiología del infarto cerebral y no volvió a presentar eventos similares. En marzo del 2019, acusó cefalea intensa, presentó vómito , estado confusional severo, y conducta infantil. Último episodio de cefalea intensa se acompañó de vómito y de severo estado confusional, acompañándose de dismnesia al referirse a sus familiares y adopción de conducta infantil que hizo pensar en un trastorno psiquiátrico al personal médico que lo atendió de emergencia. Al día siguiente tuvo consulta por consultorio externo de psiquiatría y en plena evaluación desarrolló inconciencia y sacudidas clónicas en los cuatro miembros de breves minutos de duración. En esta condición se produce su internamiento en el Hospital Nacional Dos de Mayo. Previamente no tuvo consultas de la especialidad de neurología en ningún hospital por los trastornos cognitivos y disturbios conductuales de la personalidad.

\section{Examen clínico-neurológico}

El examen clínico y por sistemas; mostró a un paciente con funciones vitales normales y la exploración por sistemas que incluyó el tórax, 
abdomen, y el uro-genital normales. La exploración neurológica mostró marcadas alteraciones en la esfera emocional, afectivamente indiferente, desinteresado por su estado de salud. Buen nivel sensorial. La exploración de la función cognitiva se determinó mediante pruebas neuropsicológicas que incluyeron el test minicognitivo de Folstein en el que obtuvo una puntuación de 16/30, la escala de inteligencia para adultos de Weschsler que incluyó el coeficiente intelectual verbal, el de ejecución, ambos promediaron un coeficiente intelectual total de 72, que calificó al paciente de estado fronterizo. Los resultados calificaron al paciente de ser poseedor de un nivel inferior en la inteligencia general; el interés al mundo circundante, la adaptación al medio ambiente, la experiencia social y el pensamiento abstracto comprometidos. En la ejecución presentó nivel inferior en la atención, memoria mecánica e inmediata, así como en la capacidad de abstracción $\mathrm{y}$ en discriminar detalles.

En el test de Machover: inestabilidad, dependencia, deseos de protección y agresividad. Finalmente en la prueba de Beck reveló depresión moderada. Las pruebas empleadas permitieron determinar las características del deterioro cognitivo. El resto de la exploración neurológica que incluyeron la valoración del lenguaje, la función práxica, los pares craneales, la función motora, coordinación axial y de los miembros, la sensibilidad superficial y profunda, y los reflejos manuales y faciales (prehensión y succión ) fueron normales. El fondo del ojo sin alteraciones (figura 1).

\section{Exámenes de laboratorio}

El hemograma, la hemoglobina, el hematocrito, la fórmula leucocitaria, el recuento plaquetario, el perfil de coagulación, la glucosa basal, el perfil hepático, los electrolitos, y los perfiles de lípidos y tiroideos fueron normales. Las pruebas serológicas para sífilis y HIV fueron negativas. Finalmente el estudio ecocardiográfico y las pruebas para detección de enfermedad autoinmune mediante anticuerpos antinucleares (ANA) y anticuerpos anticitoplasma de neutrófilos (ANCA) no reactivos.

\section{Investigación con neuroimagenes}

Los estudios con tomografía espiral a multicorte (TEM), Angio-TEM, y la resonancia magnética del encéfalo (RM) permitieron visualizar el parénquima cerebral y las características de los vasos arteriales de los sistema carotídeos y vértebro - basilares. No se pudo efectuar el estudio angiográfico digital por austeridad económica. El estudio con TEM del cerebro identificó múltiples áreas de pequeños infartos cerebrales dependientes de la circulación anterior, en estadios subagudos y crónicos a nivel de los giros frontal superior y órbito - frontal derechos con aspecto subagudo - crónico ; a nivel de la cabeza del núcleo caudado derecho y la rodilla del cuerpo calloso con aspecto subagudo ; a nivel del núcleo caudado y brazo anterior de la cápsula

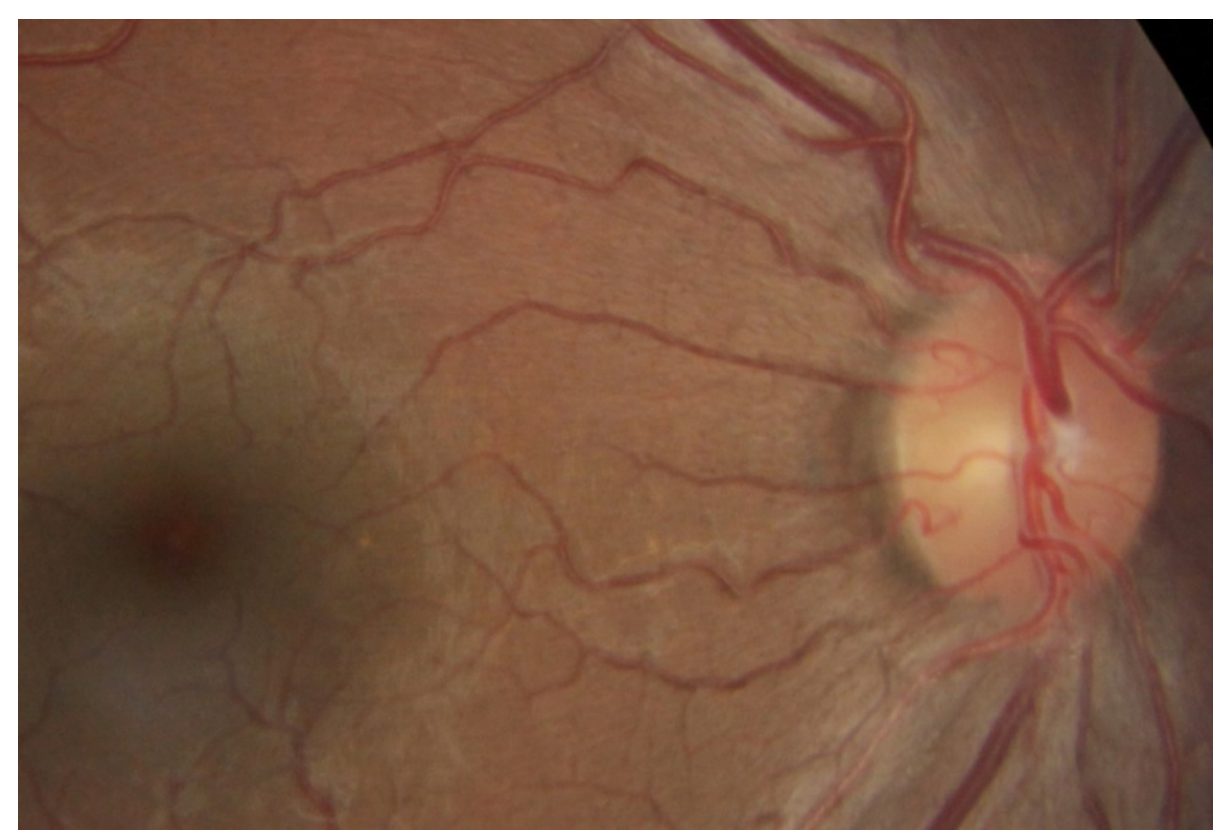

Figura 1 : Fondo del ojo sin alteraciones. 

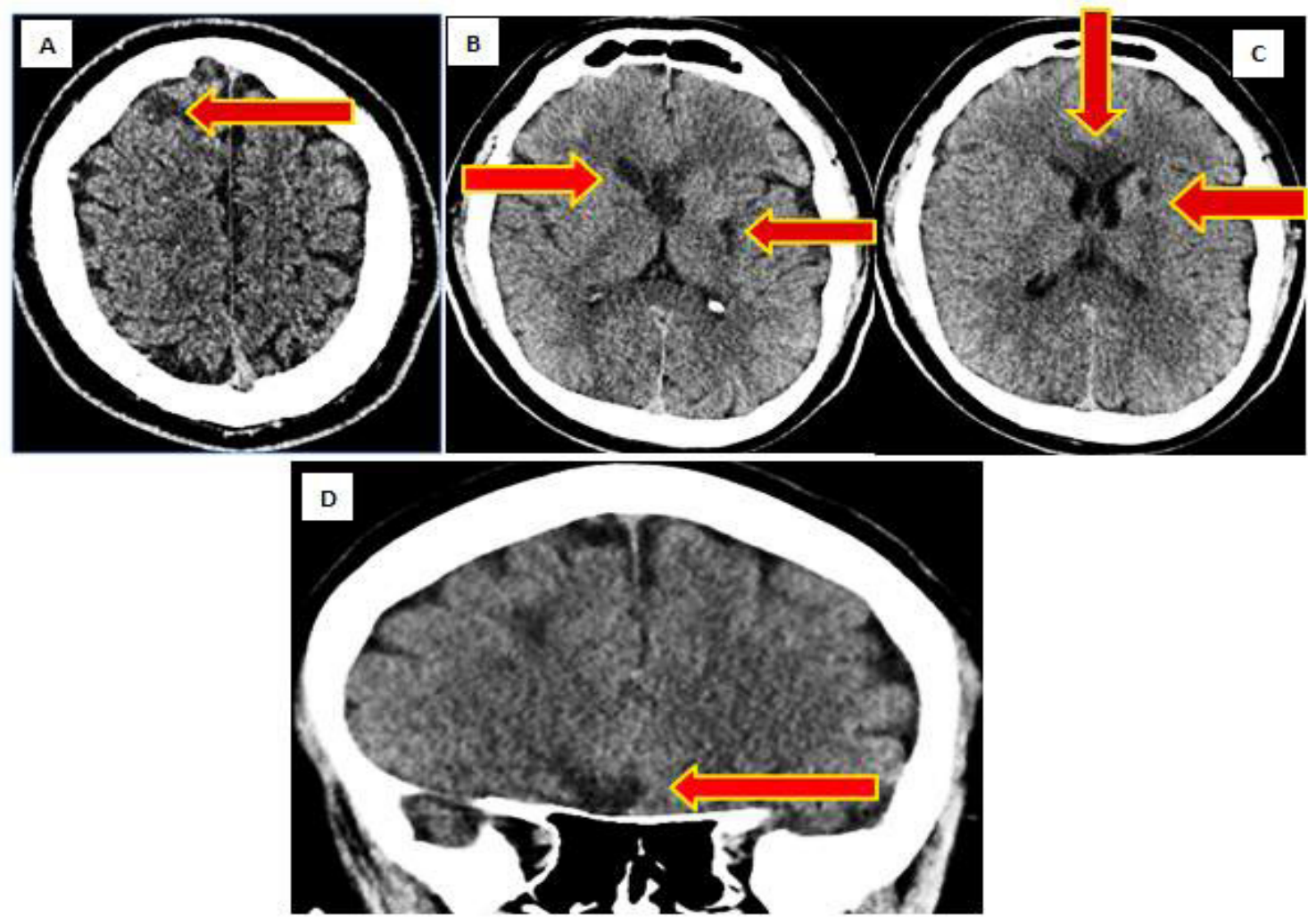

Figura 2. TEM de cerebro sin contraste, 3 cortes axiales en diferentes niveles y un corte coronal anterior que muestran múltiples infartos cerebrales dependientes de la circulación anterior en diferentes estadíos evolutivos ( subagudos y crónicos ) y niveles; frontal derecho superior en corte axial en A e inferior en D. Cabeza del núcleo caudado derecho y putamen izquierdo B y rodilla del cuerpo calloso y cabeza del núcleo caudado izquierdo en C.
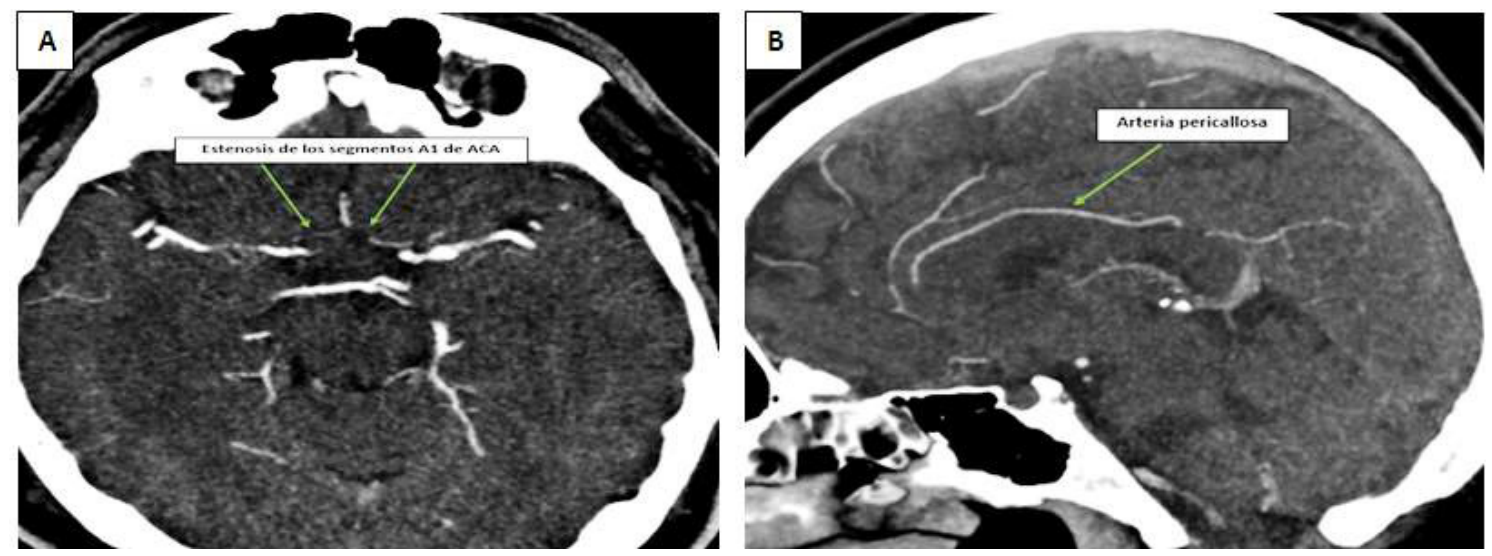

Figura 3. Estenosis severa de las arterias cerebrales anteriores (A), y visualización de las arterias pericallosas (B).

interna izquierda y en la región posterior del putamen izquierdo de aspecto crónico (figura 2). El estudio con Angio-TEM mostró estenosis severa de las arterias cerebrales anteriores, visualización de las pericallosas, presencia de aneurisma en el segmento M1 de la arteria cerebral media izquierda con estenosis proximal e inadecuada representación de la comunicante anterior. Ausencia de la comunicante posterior derecha y presencia de la arteria trigeminal (figura 3, figura 4 y figura 5).

El estudio con RM del cerebro reveló el infarto en la rodilla del cuerpo calloso en T1 sagital y los infartos precisados mediante el estudio con TEM en difusión y en las incidencias en T2 (figura 6). 


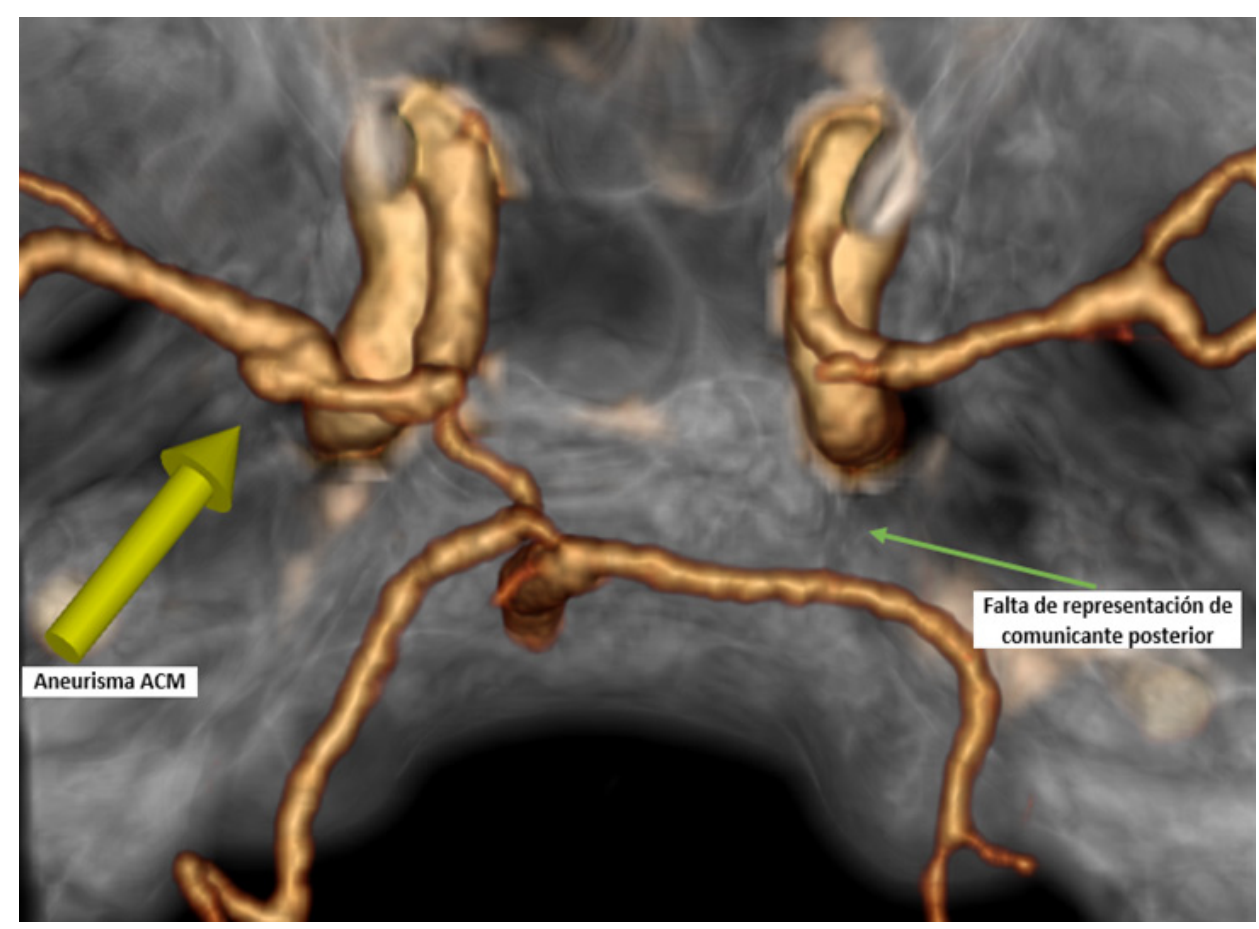

Figura 4. Aneurisma en la ACM izquierda, con estenosis proximal y ausencia de lacomunicante posterior derecha.

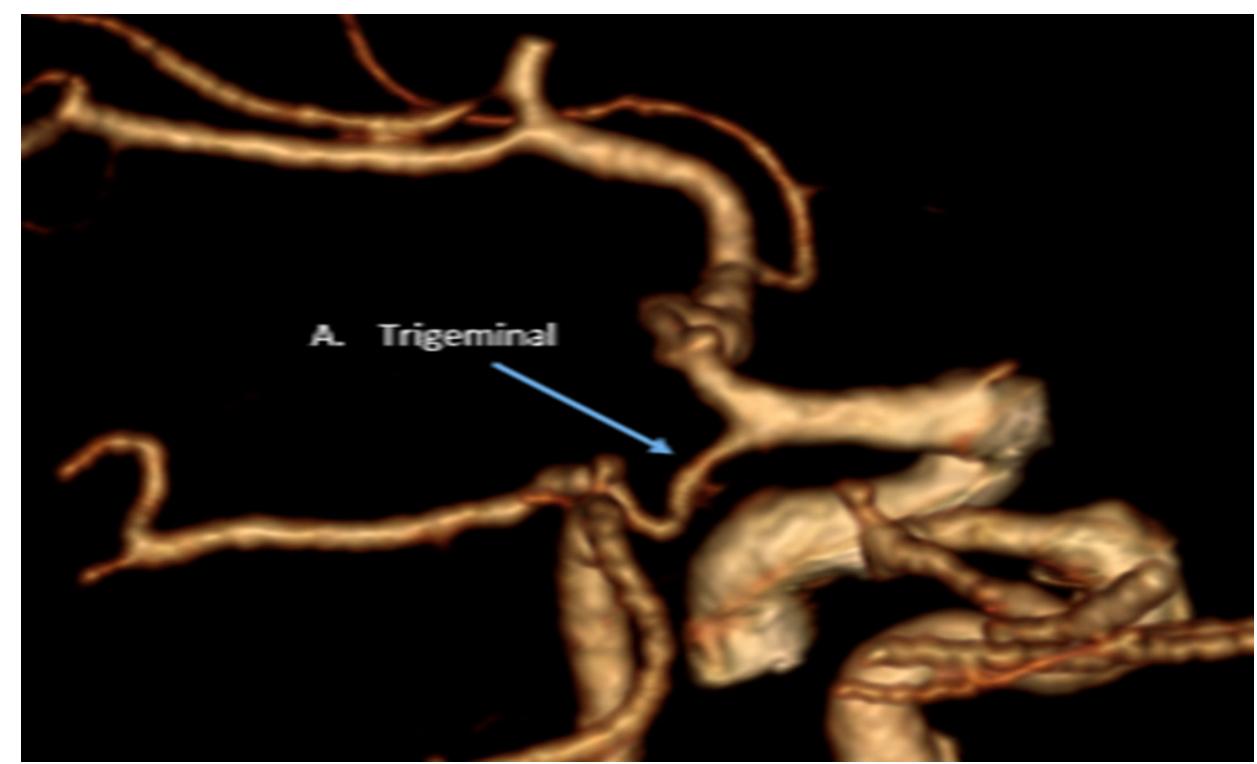

Figura 5. Presencia de la arteria trigeminal y microaneurisma de la ACM izquierda con estenosis proximal.

\section{DISCUSIÓN DEL CASO}

La enfermedad de "moyamoya" es una patología cerebrovascular oclusiva bilateral (afecta a ambos hemisferios ), caracterizada por la estenosis progresiva o por la oclusión en los extremos distales de las arterias carótidas internas, junto con una oclusión de las arterias del polígono de Willis. En casos raros, este proceso también involucra la circulación posterior incluidas las arterias cerebrales basilar y posteriores $(7,8)$. La semejanza con las volutas de humo de cigarrillo de estos pequeños vasos cuando se realiza una angiografía dió lugar al término japonés "moyamoya", que puede 


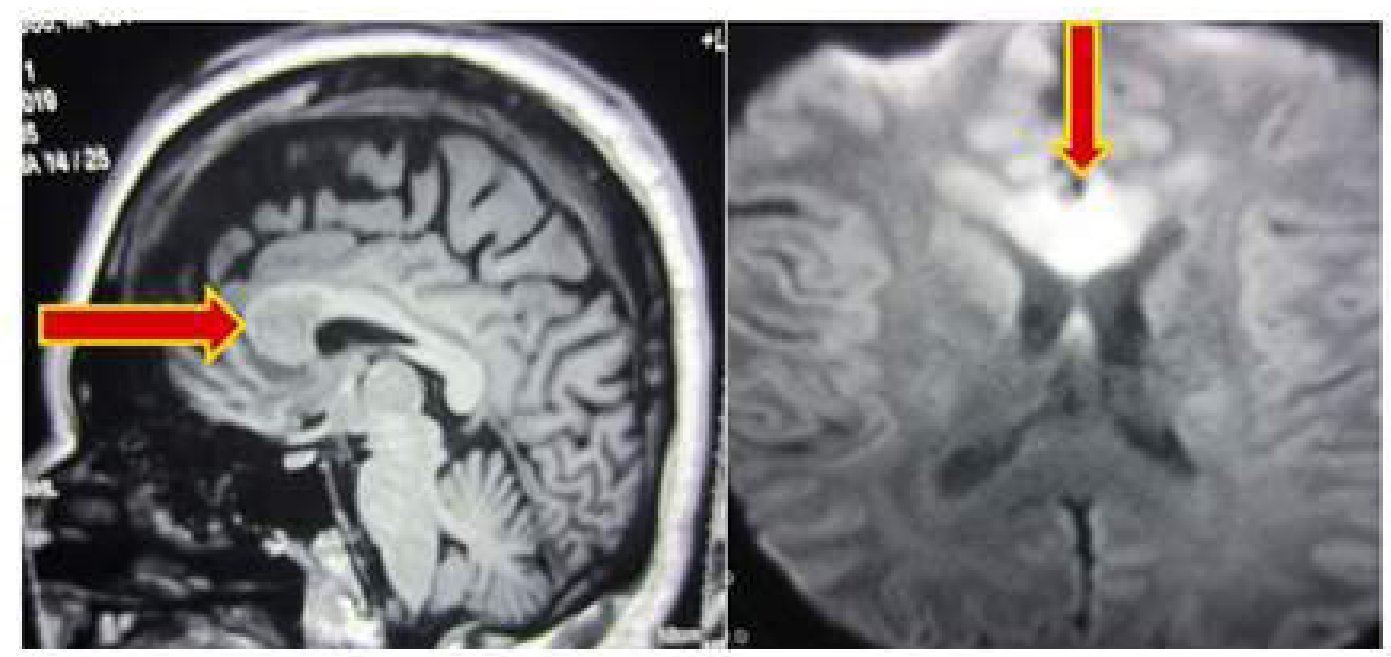

A

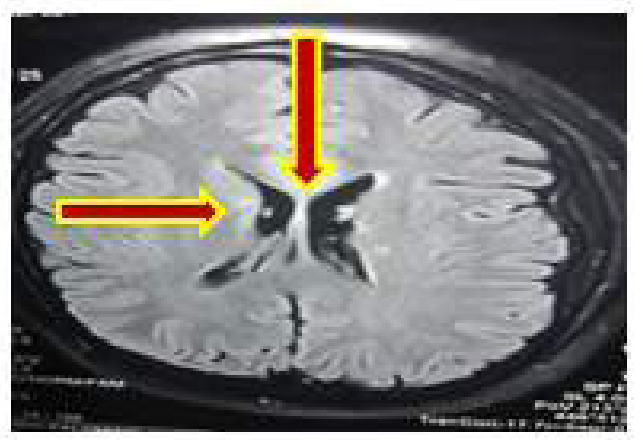

C
B

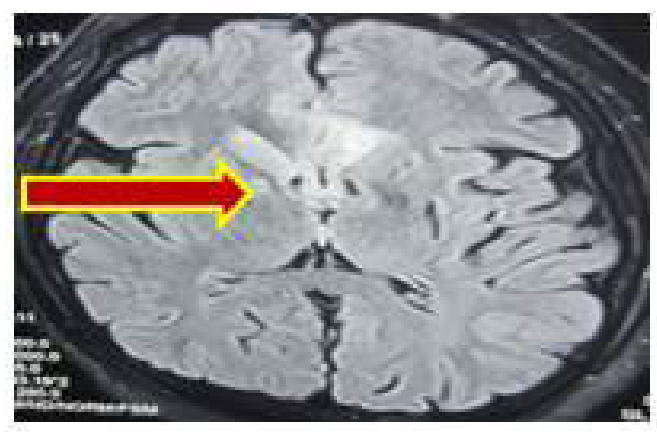

D

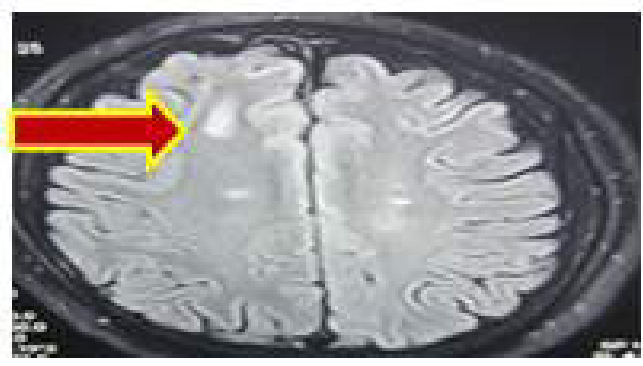

E

Figura 6. Resonancia magnetica cerebral muestra: Infarto en la rodilla del cuerpo calloso en T1 sagital en A; difusión axial en B. Difusión axial en C: infarto de la rodilla del cuerpo calloso y el caudado derecho. Infarto subcortical derecho crónico en D. Infarto frontal superior derecho en $\mathrm{E}$

traducirse como "nube de humo de cigarrillo" (9). La etiología de la enfermedad no está clara, aunque los hallazgos de una mayor incidencia en Asia oriental y el hecho de que esta patología es familiar, sugieren la implicancia de un factor genético en su patogénesis.
Recientemente se ha identificado el gen RNF213 como un gen de susceptibilidad que está presente en el $95 \%$ de pacientes con EMM familiary en el 79\% de casos esporádicos (10). En términos generales hay dos categorías etiológicas principales de síntomas: los debidos a la isquemia cerebral (ictus, 
ataques isquémicos transitorios y convulsiones) y los síntomas hemorrágicos debidos a los efectos secundarios de los mecanismos compensatorios que responden a la isquemia (hemorragias de los vasos colaterales frágiles, aneurismas relacionados al flujo y cefalea crónica por vasos transdurales dilatados). Las variaciones individuales en el grado de afectación arterial, la progresión de la estenosis , las regiones de la corteza isquémica y la respuesta a la reducción en el suministro de sangre ayudan a explicar el amplio rango de presentaciones clínicas en la EMM (11). Finalmente, un hallazgo oftalmológico ocasionalmente visto en asociación con la EMM es el denominado "síndrome de gloria de la mañana" un agrandamiento del disco óptico con anomalías retino-vasculares concomitantes. Si se observa este hallazgo, se debe considerar el diagnóstico de la enfermedad de "moyamoya " (12). En general el déficit cognitivo en esta patología se ha descrito como un trastorno neuropsicológico de origen vascular en forma de alteraciones de la memoria, atención y conducta en adultos, incluso sin evidencia neuro - radiológica de grandes ictus $(13,14)$. Nakagawara et al., indicaron que esta condición se da como consecuencia de disfunción cerebral asociada con una alteración hemodinámica en los lóbulos frontales mediales (incluyendo el giro cingulado anterior ) medida con tomografía con emisión de positrones (SPECT) (15).

Los datos de nuestro paciente, adulto, sin antecedentes de ascendencia asiática,con ausencia en los exámenes auxiliares de hallazgos compatibles con enfermedad ateroesclerótica, infecciosa o de carácter autoinmune, la cefalea crónica por más de 15 años, el progresivo deterioro cognitivo con afectación de la atención, memoria, y la concentración acompañadas de severos disturbios afectivos como indiferencia personal de su estado y el entorno desarrollados en los últimos siete años, un episodio de desorden vascular isquémico acontecido cinco años atrás y finalmente el último episodio de infarto de la rodilla del cuerpo calloso que ocasionó severo estado confusional e intensificó el estado demencial, refrendados por los resultados en el estudio con imágenes que muestran los infartos múltiples, las severas estenosis de las arterias cerebrales anteriores, el aneurisma en el inicio de la cerebral media izquierda con estenosis proximal, son criterios para el diagnóstico de enfermedad de Moyamoya, estado crónico de la enfermedad.
En Perú se han descrito cinco observaciones clínicas complementadas con angiografía con sustracción digital en las que reportan la imagen característica de "nubes de humo" en pacientes adultos que presentaron hemorragia subaracnoidea recidivante (16), síndrome coreico asociado a hematoma subagudo tálamo capsular derecho (17) y un paciente que desarrolla síndrome confusional con manifestaciones de sígnos mesencefálicos, ocasionado por hemorragia talámica derecha (18). Frecuentemente es difícil diagnosticar la EMM en pacientes que se encuentran en la etapa inicial de la enfermedad, es decir, cuando la red vascular no es tan evidente; en estas condiciones el empleo de la resonancia magnética de alta resolución, que permite identificar características de la pared del vaso y precisar la etiología de las diferentes causas de estenosis características patológicas concretas de la pared del vaso es recomendable (19).

\section{CONCLUSIONES}

Consideramos que la presentación del caso nos conduce a contribuir con mayor profundidad sobre esta enfermedad que afecta a la población infantil y adulta con mayor incidencia en las poblaciones asiáticas, sin embargo, su ocurrencia en nuestro medio no debe ser subestimada. El espectro clínico de esta vasculopatía es amplio y el deterioro cognitivo puede ser el de mayor relevancia. De allí la importancia del empleo de las pruebas neuropsicológicas en niños y en adultos que presentara este trastorno. Este es el primer reporte de la EMM en un adulto, en nuestro medio, que desarrolló infartosmúltiples y que ocasionó severa discapacidad cognitiva crónica progresiva.

\section{Correspondencia}

Marco A. Castañeda Reyna

Servicio de Neurología, Hospital Nacional Dos de Mayo

Jr. Moquegua 3655. San Martín de Porres, Lima, Perú Teléfono : 51987185856

Correo electrónico: marcocastanedareyna@gmail. com

\section{REFERENCIAS BIBLIOGRAFÍCAS}

1. Takeuchi K, Shimizu K. Hypogenesis of bilateral internal carotid arteries. No To Shinkei. 1957; 9: 3743.

2. Baba T, Houkin K, Kuroda S. Novel epidemiological 
features of moyamoya disease. J Neurol Neurosurg Psychiatry. 2008;79: 900-904.

3. Mineharu Y, Takenaka K, Yamakawa H. Inheritance pattern of familial moyamoya disease: autosomal dominant mode and genomic imprinting. J Neurol Neurosurg Psychiatry. 2006; 77(9): 1025-1029.

4. Ikesaki K, Han DH, Kahuano T, Kinukahua N, Fukui M. A clinical comparison of definite moyamoya between South Corea and Japan. Stroke. 1997; 28: 2513-7.

5. Scott RM, Smith JL, Roberson RL. Long-term outcome in children with moyamoya syndrome after cranial revascularization by pial synangiosis. J Neurosurg. 2004; 100: 142-9.

6. Ju LB, Shang Q, Shi ZY, Wang MQ, Shang D. High resolution magnetic resonance imaging of Moyamoya disease. Chin Med J (Engl). 2015; 128(23): 32313237. doi: 10.4103/0366-6999.170257.

7. Suzuki J, Kodama N. Moyamoya disease-a review. Stroke. 1983; 14(1):104-109.

8. Janda PH, Belew GJ, Veerapan V. Moyamoya disease: case report and literature review. J Am Osteopath Assoc. 2009;109(10):547-553.

9. Susuki J, Takaku A. Cerebrovascular "moyamoya" disease showing abnormal net-like vessels in base of brain. Arch Neurol. 1969; 20(3):288-99.

10. Liu W, Morito D, Takashima S. Identification RNF 213 as a Susceptibility gene for moyamoya disease and its possible role in vascular development. PLoS One. 2011;6(7):e22542. doi: 10.1371/journal. pone. 0022542

11. Scott RM, Smith ER. Moyamoya disease and moyamoya syndrome. N Engl J Med. 2009; 360: 1226-37.
12. Massaro M, Thorarensen O, Liu GT, Maguire AM, Zimmerman RA, Brodsky MC. Morning glory disc anomaly and moyamoya vessels. Arch Opthalmol. 1998; 116: 253-254.

13. Araki Y, Tagaki Y, Ueda K, Ubukata S. Cognitive funtions the patient with adult moyamoya disease. J Cerebrovasc Dis. 2015; 23: 17891794.

14. Karzmark P, Zeifert PD, Bell-Stephens TE. Neurocognitive impairment in adults with moyamoya disease without stroke. Neurosurgery. 2012; 70: 634-638.

15. Nakawahara J, Osato T, Kamiyama K. Diagnostic imaging of higher brain dysfuntion in patients with adult moyamoya disease using statistical imaging analysis for (123I) iomazenil single photon emission computed tomography. Neurol Med Chir (Tokio). 2012; 52: 318-26.

16. Rosell A, Rocca U, Giraldo E. Enfermedad de Moya Moya en el Perú. Rev Cuerpo Méd. 1994;14(2):3843.

17. Villafuerte M, Vélez M, Flores M, Torres L. Un caso de enfermedad de Moya Moya con movimientos coreicos. Rev Per Neurol. 2012 ; 12 (1): 21-25.

18. Ramírez-Quiñones J, Barrientos-Imán D, Calle-La Rosa P, Ecos-Quispe R. Enfermedad de Moyamoya: reporte de un caso. Revista de Neuro-Psiquiatría. 2015; 78(3): 165-170.

19. Yuan M, Liu ZQ, Wang ZQ, Li B, Xu LJ, Xiao XL. High resolution MR imaging of the arterial wall in moyamoya disease. Neurosci Lett. 2015; 584:77-82.

Recibido: 22/10/2019 Aceptado: 19/11/2019 